\title{
Stroke Research Priorities for the Next Decade - A Representative View of the European Scientific Community
}

\author{
Summary of Stroke Research Workshop, Brussels, October 25, 2005
}

\author{
Stephen Meairs Nils Wahlgren Ulrich Dirnagl Olle Lindvall Peter Rothwell \\ Jean-Claude Baron Konstantin Hossmann Britta Engelhardt José Ferro James McCulloch \\ Markku Kaste Matthias Endres Jari Koistinaho Anna Planas Denis Vivien Rick Dijkhuizen \\ Anna Czlonkowska Arne Hagen Alan Evans Gennaro De Libero Zoltan Nagy \\ Daiva Rastenyte Jürgen Reess Antoni Davalos Gian Luigi Lenzi Pierre Amarenco \\ Michael Hennerici
}

About one million strokes occur each year in the European Union [1]. Indeed, about $25 \%$ of men and $20 \%$ of women can expect to suffer a stroke if they live to be 85 years old. As a cause of death worldwide, stroke is second only to coronary heart disease [2].

Although stroke is a major cause of death, mortality data underestimate its true burden. This is chronic disability. Since stroke causes disability more often than death, stroke patients frequently require long hospital stays followed by ongoing support in the community, or nursing home care. Stroke is consequently a major drain on health care funding. Stroke is the number one cause of disability in the European Union.

The total incidence of stroke is projected to increase considerably over the next two decades. This is because of the rapid increase in the elderly population. It is predicted that stroke will account for $6.2 \%$ of the total burden of illness in 2020 [3]. Thus, without more effective strategies for the prevention, treatment, and rehabilitation of stroke, the cost of this disease will increase dramatically.

The European Commission hosted a European Stroke Workshop in Brussels on October 25, 2005, gathering top European experts in clinical stroke management, basic science, stroke research and industry. The primary objective of the workshop was to exchange views on the topic of stroke and to identify research activities that could potentially result in major advances in the areas of stroke prevention, treatment and recovery. These research priorities should address the most pressing scientific, clinical and industrial needs in the field of stroke. Participants agreed that answers to the questions posed by these research priorities must significantly help in reducing the stroke burden to the European Union. The following is a summary of the ideas that were presented, discussed and agreed upon during the Stroke Workshop. Several of the key topics, particularly those in basic science stroke research and imaging, span prevention, therapy and recovery, thus conceivably making contributions to multiple areas of stroke management.

\section{Achievements, Opportunities and Future Goals in Stroke Research}

The last 25 years have seen remarkable progress in the understanding of the pathophysiology of ischemic stroke. European research centers have been and remain at the

\section{KARGER}

Fax +4161306 1234 E-Mail karger@karger.ch www.karger.com
(C) 2006 S. Karger AG, Basel

$1015-9770 / 06 / 0223-0075 \$ 23.50 / 0$

Accessible online at:

www.karger.com/ced
Dr. Stephen Meairs

Neurologische Klinik, Ruprecht-Karls-Universität Heidelberg

Theodor-Kutzer-Ufer, DE-68135 Mannheim (Germany)

Tel. +49 621383 3550, Fax +496213833807

E-Mail meairs@neuro.ma.uni-heidelberg.de 
forefront of basic and clinical stroke research, having made seminal contributions to stroke pathophysiology and clinical management of stroke.

This 'first wave' of stroke research produced relevant animal models of cerebral ischemia that allowed the identification of mechanisms that contribute to tissue damage. It is now recognized that the critical reduction of blood supply to an area of the brain leads to a complex cascade of events, which evolves over time and space. This knowledge has opened the possibility to intercept this cascade therapeutically. In animal models of stroke, brain tissue can be successfully protected from damage. However, attempts to translate these findings into clinical practice have not yet been successful, although recently positive results have been obtained in clinical trials with the free radical scavenger NXY-059.

Only recently has it been understood that the capacity of the nervous system for regeneration, including the formation of neurons in adulthood, is greater than previously believed. This has opened new horizons for the treatment of stroke utilizing strategies to induce the brain's own mechanisms of protection and regeneration. Moreover, this has led to treatment with stem cells of various sources, which have successfully restored brain function after experimental stroke.

Impressive developments have occurred in stroke trial methodology and in new treatment approaches. Aspirin and other antiplatelet agents are now used in secondary stroke prevention, as well as anticoagulants for the subgroup of patients who experience a stroke from a cardiac source of emboli. Blood pressure lowering after stroke and transient ischemic attack (TIA) helps to prevent recurrent stroke and cardiac events. Accumulating evidence also suggests that statins have a prominent role in secondary prevention of stroke. Endarterectomy for symptomatic high-grade stenosis of the carotid artery has proven to protect against new ipsilateral stroke.

Experiences from research on interventions to combat acute stroke are mixed. We now know that only a short therapeutic window of a few hours is available for reopening the blood supply to the ischemic brain. This has resulted in calls upon programs to increase public awareness and to improve the prompt availability of stroke care through well-organized services. Tissue plasminogen activator ( $t-P A)$ is the only drug approved by the EMEA for intravenous thrombolysis of acute ischemic stroke.

The establishment of thrombolysis as the first successful therapeutic strategy in acute stroke, the development of novel noninvasive brain imaging strategies, as well as advances in trial design and hyperacute patient recruitment have raised the hope that we will be able to protect the brains of patients with stroke in the near future. Current trials take advantage of second-generation thrombolytics with lesser side effects on the vasculature and brain parenchyma. Moreover, imaging technologies like perfusion- and diffusion-weighted MRI allow the identification of tissue at risk and can be used to identify patients in whom brain protective therapy is likely to be successful. A 'second wave' of combined efforts in basic and clinical stroke research can now capitalize on these promising developments.

European research is in an excellent position to make significant advances in the field of stroke. Core skills of established centers of excellence in basic research provide ample opportunities when combined with the EU ethos for collaboration and interdisciplinarity. Collaborative efforts of European stroke researchers can draw on already existing networks, established in large part by EU funding.

The Stroke Workshop participants identified the following research fields in which a European research offensive can be highly successful on an immediate to intermediate time scale.

\section{Cerebral Reperfusion}

$\mathrm{t}$-PA is the only drug that is able to improve the outcome of patients with ischemic stroke. By enhancing the endogenous formation of plasmin from plasminogen, the blood clot occluding a cerebral vessel can be dissolved by disruption of fibrin. Although the reperfusion strategy has proven to be a successful concept for treating acute stroke, there are still considerable scientific opportunities to further develop this approach and to achieve improved benefit for patients.

One experience from the use of t-PA in acute ischemic stroke is that a substantial proportion of the patients do not improve, or only mildly improve, after administration of the drug. Identification of this lack of treatment response and development of an alternative strategy to overcome this problem is a scientific priority. Accumulating evidence is already available for such approaches, but these need to be refined and evaluated in an extended clinical setting.

One possible explanation for an insufficient treatment response is that the dose of $\mathrm{t}-\mathrm{PA}$, restricted by safety concerns, is insufficient to dissolve particularly dense thromboembolic clots. Increased knowledge of the significance 
of variations in the size, composition and location of the blood clot would be useful for advancing this approach. Fibrinolytic gene polymorphism may be another factor explaining individual variability in treatment response. Mapping of such variability and development of new pharmacological agents which may enhance the fibrinolytic response of t-PA are interesting challenges. It has also been suggested that observations of neurotoxic effects in animal stroke models of t-PA may be relevant for the clinical response in human stroke patients and restrict the beneficial effects of reperfusion.

There may be additional ways to enhance the reperfusion concept. Combinations of intravenous thrombolysis with intra-arterial approaches are promising and need clinical large-scale evaluation. Ultrasound may facilitate fibrinolysis. The effect of thrombolysis in combination with potential neuroprotective drugs needs further exploration. Further research into vascular biology mechanisms may provide enhanced understanding of the importance of the blood brain barrier during reperfusion and identify markers that can predict hemorrhage induced by thrombolytics.

- Not only do relatively few patients benefit from t-PA because of the narrow time window, its neurotoxic activities may also limit its clinical potential. Novel thrombolytic agents need to be developed and their effectiveness tested to overcome this hurdle.

- Intra-arterial interventions used for locally applied pharmacological agents or mechanical impact on the occlusion may be used in case of treatment failure or in patients for whom predictions may indicate that intravenous thrombolysis will be less successful.

- Ultrasound can induce microstreams within the clot resulting in in-depth distribution of t-PA to facilitate lysis. Ultrasound in combination with novel microand nanoparticles offers further therapeutic potentials for stroke treatment, i.e. thrombolysis, targeted drug delivery and increased collateral flow to the ischemic penumbra. Rapid technological progress is being made in several European centers in both ultrasound imaging and therapeutic applications of ultrasound.

Cerebral bioeffects of these new technologies, however, are not well understood, and appropriate in vitro and in vivo models for investigating these bioeffects are lacking. There is considerable fragmentation in European efforts and standardization in this area is lacking.

\section{Research Priorities - Cerebral Reperfusion}

- Identify and test nonneurotoxic thrombolytics, understand mechanisms by which current thrombolytics damage vessels and neurons.

- Determine the most optimal approaches of ultrasound facilitation of clot lysis. Evaluate methods for delivering thrombolytic agents using microbubble targeting. Obtain knowledge on the bioeffects of therapeutic ultrasound applications for treatment of stroke to optimize therapeutic goals and limit adverse side effects.

- Organize clinical trials of intra-arterial approaches integrated with intravenous thrombolysis.

- Develop methods to identify individual variability in response to thromboembolic occlusion in cerebral arteries.

\section{Optimizing the Delivery of Stroke Care}

Prompt therapy of stroke is of fundamental importance for limiting the acute lesion ('time is brain'). Early identification of stroke patients, optimization of initial medical management, and techniques for early evaluation of symptoms, have a high likelihood of being successful and need systematic evaluation. General treatment of stroke including blood pressure, blood glucose and fever is based on consensus of experts, not on scientific evidence. We need reliable evidence to guide acute stroke care starting from prehospital emergency medical services continuing through emergency wards to stroke unit care.

The positive outcomes of clinical trials documenting the effect of stroke units, thrombolysis and recently hemostasis have not been followed by a consistent implementation into clinical practice. An extensive network of more than 350 acute stroke centers with focus in the European Union provides a strong support for implementation services with accumulation of data from around 7,000 treatments in the Safe Implementation of Thrombolysis in Stroke database. Updated information on the use of stroke units are also required, since the data bank of the Stroke Unit Trialists' Collaboration is based upon studies carried out 10-20 years ago. Modern stroke unit care is something totally different and its efficacy needs to be scientifically evaluated. Success is unlikely unless scientific methods are used to explore factors that delay and factors that facilitate implementation of evidence-based treatments. State-of-the art stroke unit care must also be evaluated and information regarding its efficacy distrib- 
uted and implemented widely to decrease human and economic burden of stroke.

- Broad-scale evaluation of different levels of stroke care in academic settings, rural areas, prehospital care in EU member states, of factors which may delay or facilitate safe broad implementation of thrombolysis and other stroke interventions may create a basis for bringing effective stroke interventions to patients.

- By developing new approaches for prehospital management, the time between onset of symptoms and initiation of therapy can be shortened and patients may be better selected for therapy. Early biomarkers may also help in patient selection.

- The development of novel ultrasound imaging equipment or other promising technologies for stroke detection may allow mobile stroke imaging. When linked to advanced telemedicine systems, such diagnostic equipment could provide information to guide prehospital thrombolysis and neuroprotection.

\section{Research Priorities - Optimizing the Delivery of Stroke Care}

- Produce new data on optimal care of acute stroke patients and identify factors that may delay and factors that may facilitate broad implementation of evidencebased stroke interventions.

- Identify methods for optimal hyperacute, prehospital management of stroke and subject them to clinical evaluation.

- Link new mobile brain imaging equipment for prehospital diagnosis of stroke with advanced telemedicine to provide new strategies for prehospital therapy of stroke.

\section{Brain Protection}

The treatment window for thrombolysis is so narrow that only a minority of patients arrive early enough in hospitals. Neuroprotecting agents could have a longer time window for treatment and less severe side effects. They could not only add to the efficacy of thrombolysis and also reduce the risk involved in it, but also be effective as a stand-alone treatment or be combined with other brain protective treatments, including hypothermia, in patients not eligible for thrombolysis. Recent experimental research has identified many basic mechanisms involved in the progress of ischemic penumbra in brain infarction.
This opens new possibilities for more effective therapies and also for combination therapies. The same holds true for agents enhancing neuronal regeneration after experimental stroke. These observations may open totally new treatment strategies for enhanced neuronal recovery, reorganization and improved neuroplasticity. Success in the field of enhanced neurorecovery in basic research will translate to patient treatment and also could widen the time window of acute stroke therapy to allow many more acute stroke patients to benefit from these breakthroughs. Recent advances in understanding the signaling mechanisms of ischemic damage and endogenous neuroprotection set the stage for a focused effort to close gaps in our knowledge, and to develop therapeutic strategies from this knowledge.

- We need to protect not only neurons, but the whole network of brain cells (astrocytes, oligodendrocytes, microglia, etc.) affected by focal cerebral ischemia. Therefore, we will need to focus more on white matter injury. Surprisingly, white matter injury is rarely addressed in current stroke research, despite its relevance for the potential of the neurons to recover, and for a particular type of cerebral ischemic syndrome, vascular dementia.

- It is becoming clear that a successful treatment of stroke will need to block various mechanisms of damage simultaneously, hence the need for combination therapy. The preclinical establishment of combination therapy is demanding, and only feasible as a joint effort of many laboratories (large multicenter preclinical studies). EU scientists also need to establish common criteria for performing such research and to assure quality. Establishing SOPs and rules for conducting and reporting experimental stroke studies would also facilitate systematic reviews, and help to close the gap between clinical and experimental stroke research.

- An important message of recent stroke research is that to harness a full therapeutic potential; brain protective strategies should not only focus on the brain. Specifically, brain-cardiovascular and neural-immune interactions have been identified as highly relevant for tissue damage as well as repair after a stroke. For example, infection is the most important complication after a stroke, as well as the number one cause of death after the first $24 \mathrm{~h}$ after symptom onset.

- There is a great need for clinical breakthroughs for neuroprotective and neurorecovery-enhancing treatments for acute stroke treatment. Based on progress in basic research we now understand much more about 
ischemic cell death. This information opens a gate for successful translation to stroke patient treatment and now is the time to take the challenge.

\section{Research Priorities - Brain Protection}

- Identify strategies to protect not only neurons, but also vascular and glial cells after focal cerebral ischemia. Focus more on white matter injury, small vessel disease and address vascular dementia.

- Conduct multinational preclinical trials on combination therapy. Establish SOPs, 'good laboratory practice', and reporting standards for experimental stroke research.

- Identify and understand cardiovascular-brain and neuralimmune interactions after stroke, develop therapeutic strategies.

- Evaluate neuroprotective and neurorecovery-enhancing treatments as stand-alone and combined therapies in clinical trials.

\section{Recovery after Stroke}

Based on advances in stem cell biology there is potential for therapy of chronic and acute brain disorders such as stroke. However, a number of important issues need to be explored before clinical trials of stem cell therapy in stroke patients can be considered.

- Transplanted cells of different sources have induced incomplete recovery in animals and humans affected with stroke, probably through trophic support. In a future clinical application, the grafted stem cells most probably have to be of human origin. Research should now focus on testing various types of stem cells (embryonic stem cells, stem cells from fetal and adult brain, and from other tissues) in animal models of stroke for their ability to generate and replace those particular neurons which have died, to remyelinate the demyelinated axons, and to repair the damaged neural circuitries. Only cells which can act through either some or all of these mechanisms, directly or after predifferentiation in culture, are likely to induce improvement of major therapeutic value in a clinical setting. It is also necessary to learn how to control the proliferation and differentiation of the stem cells into the specific cellular phenotypes.

- Behavioral recovery after transplantation of the most promising cell types has to be optimized in animal models of stroke. The grafted cells should be able to survive, integrate and differentiate in large numbers. The time window after the stroke when transplantation will lead to maximum restitution of neural circuitries and functional recovery should be determined. It will be important to be able to influence the pathological tissue environment after stroke, including inflammatory and immune reactions, to allow for efficient repair. One valuable strategy to promote recovery will probably be to combine stem cell and gene therapy. The stem cells could be genetically modified to improve their survival and differentiation, or genes could be delivered directly into the stroke-damaged brain to improve the migration and functional integration of the grafted cells.

- Stroke-damaged adult rodent brain has some capacity for neuronal replacement from its own neural stem cells. During several months after stroke, neural stem cells in the subventricular zone, lining the lateral ventricles, generate new striatal neurons which migrate to the damaged area. Stroke-induced neurogenesis is maintained in the aged brain. It is now important to establish whether endogenous neurogenesis contributes to functional recovery after stroke, and if it occurs in humans. Also, it needs to be determined whether endogenous neural stem cells can be triggered to produce cortical neurons, which are needed for improvement in most stroke-damaged brains. Strategies to increase survival of new neurons and stimulate their incorporation into reorganizing neural circuitries should be developed.

- The development of imaging techniques for assessment of grafted and endogenous stem cell survival, migration, and function after stroke is necessary for the advancement of this research field. Some promising data are already available using magnetic resonance imaging.

- Safety issues are of major importance. Strategies to eliminate the risk for tumor formation, in particular, from embryonic stem cells have to be developed. Prior to clinical application, other adverse effects of stem cell therapy in stroke, such as involuntary and inappropriate movements and sensory dysfunction, should be carefully screened for in animals.

- There is a need to define which subset of stroke patients will be suitable for stem cell therapy. This will depend both on stroke pathology, location, and symptomatology in patients, as well as on which specific neuron types can be generated from stem cells. 


\section{Research Priorities - Recovery after Stroke}

- Test various types of stem cells in animal models of stroke for their ability to generate and replace neurons which have died, to remyelinate the demyelinated axons, and to repair damaged neural circuitries.

- Explore contribution of endogenous neurogenesis to functional recovery after stroke. Learn how to trigger production of cortical neurons and stimulate integration into neural circuitries.

- Combine stem cell and gene therapy for stroke recovery. Stem cells could be genetically modified to improve their survival and differentiation, or genes could be delivered directly into the stroke-damaged brain to improve the migration and functional integration of the grafted cells.

- Further develop imaging techniques for assessment of grafted and endogenous stem cell survival, migration, and function after stroke.

\section{Cerebrovascular Biology}

Stroke is a vascular disease. Clinically successful stroke treatment and prevention modalities are thrombolysis and platelet inhibition, i.e. vascular approaches. The physiology and pathophysiology of the neurovascular unit, the complex network of endothelial, smooth muscle, glial, and neuronal interactions, needs to be understood to devise new preventive as well as protective strategies. An interdisciplinary effort of neuroscientists, vascular biologists and neurologists is clearly required.

- Our basic understanding of vascular development and vascular interfaces has greatly improved over the last decade. In contrast, our knowledge on the regulation of blood-brain barrier (BBB) function and the neurovascular unit is still behind. This knowledge needs to be expanded and integrated, extended and applied to stroke pathology. Barrier characteristics of brain endothelial cells are rapidly lost in stroke. We know that maintenance of the BBB endothelium depends on continued crosstalk with the CNS microenvironment, but the cellular and molecular mechanisms involved in this maintenance are unknown. There is evidence that improvement in clinical outcome after stroke could be achieved by maintaining $\mathrm{BBB}$ integrity and/or accelerated reestablishment of BBB function.

- Only recently have we come to understand that vessel formation and brain cell development are closely linked. Neoangiogenesis is tightly linked to neuroneogenesis, and the vasculature can provide scaffolds for neuronal migration. It is likely that these physiological processes are also highly relevant after brain damage, e.g. after a stroke. Interdisciplinary work of neuroscientists, stem cell biologists, and vascular biologists can clarify this issue.

- Recruitment of cells of the innate and adaptive immune system contributes to stroke pathogenesis. However, the sequence of molecular steps involved in the recruitment of different leukocyte subpopulations across the BBB is not completely understood.

- Over the last 20 years an impressive repertoire of small animal stroke models has been developed. However, these efforts have almost exclusively focused on large vessel occlusion. Models to study small vessel disease are needed to expand our understanding of these important clinical entities.

\section{Research Priorities - Cerebrovascular Biology}

- Perform research on the molecular and cellular biology of the BBB to understand the regulation of BBB characteristics in CNS endothelium as a prerequisite to understand the pathophysiology of the CNS microcirculation during stroke.

- Understand the cellular and molecular mechanisms with which endothelial cells of the BBB react to focal cerebral ischemia, identify targets to protect these cells, the $\mathrm{BBB}$, and the neurovascular unit against damage. Define markers of BBB disruption, and markers that can predict hemorrhage induced by thrombolytics.

- Understand the sequence of molecular traffic signals involved in leukocyte recruitment across the BBB in order to specifically prevent the migration of pathogenic leukocytes into the CNS, while at the same time maintaining the recruitment of potential repair cells.

- Understand and target angiogenesis, vasculogenesis, arteriogenesis after (or before) a stroke. Study the interaction of angiogenesis and neurogenesis, in particular after stroke.

- Develop small animal models for lacunar stroke. Investigate mechanisms of damage as they relate to nonlarge vessel occlusion. 


\section{Stroke Imaging}

The recent advances in morphological, functional, and molecular brain imaging have greatly contributed to the progress in understanding stroke pathophysiology, as well as in establishing successful treatment strategies. Approaches have been developed to stratify stroke patients for treatment (e.g. diffusion/perfusion mismatch), to noninvasively assess the brain circuitry involved in functional recovery (e.g. fMRI), and to monitor novel treatment strategies, such as cell therapy (e.g. MION label MR). However, the potential of these techniques has to be further developed, and the underlying concepts need to be validated before patients can benefit from these advances on a broader scale.

- The penumbra concept is an important breakthrough in stroke research. Novel imaging techniques now allow characterization of a 'penumbra' in individual patients. However, each approach has a different underlying concept of 'penumbra'.

- Not only relevant to stroke, but also to other acute and chronic brain disorders is the urgent need to develop $\mathrm{MR}, \mathrm{CT}, \mathrm{PET}$, ultrasound, and optical imaging techniques to study: gene expression at the transcriptional level; protein expression; protein-protein interaction; cell homing, invasion, and migration, as well as differentiation; functional activity, neural pathways and networks, plasticity and reorganization.

\section{Research Priorities - Stroke Imaging}

- Understand what the underlying pathophysiology is that produces a 'penumbra' when PET, MR, ultrasound or CT measures it. Validate the underlying concept with invasive methods in experimental models.

- European stroke researchers need to be integrated and team up with networks of scientists in which new imaging methodologies are being developed and validated, and contribute stroke specific aspects and models.

- Develop new approaches for molecular imaging. Use imaging to better understand lesion maturation and progression and clinical deterioration, and to monitor the effect of treatments.

\section{Stroke Prevention}

Specific research on stroke prevention is clearly warranted. High-quality studies in this field have not been undertaken due to the complex nature of heterogeneous stroke syndromes with different underlying pathophysiologies. Likewise, there has been a notable lack of translation of important developments in imaging and vascular biology into stroke prevention trials, particularly in studies of the elderly. As atherosclerosis, hypertension and diabetes are important risk factors for stroke, continued support of research in these areas is also needed.

The identification of rare, Mendelian forms of stroke has greatly benefited our understanding of the common, multifactorial forms of stroke, because they allow us to identify key pathways that lead to a stroke, which are likely to be involved also in the development of other forms of stroke. Large-scale European interdisciplinary efforts of molecular geneticists, cellular biologists, physiologists, and clinicians are needed to further our understanding of genetic susceptibility to stroke, as well as to the genetic influence on acute stroke pathology as well as recovery. Such knowledge should significantly contribute to further development of stroke prevention strategies.

Prevention research in this area should be targeted to:

- determine the most effective preventive strategy during the high-risk acute phase after a TIA or minor stroke.

- determine overall prognosis of particular clinical syndromes, such as TIA and stroke associated with large artery atherosclerosis in the posterior circulation, so that the need for treatment can be properly determined and treatment trials planned.

- predict the risk of stroke in individuals with syndromes, such as asymptomatic carotid stenosis or recent TIA, so that potentially risky or costly treatments can be targeted at those individuals who are most likely to benefit.

- investigate the potential of screening the elderly 'primary prevention' population to identify individuals who are at high risk of stroke.

\section{Research Priorities - Stroke Prevention}

- Novel risk factors for stroke should be identified. Moreover, we must learn how existing risk factors differ by subtype.

- Large networks should be developed to achieve the sample sizes necessary to study this highly heterogeneous clinical syndrome.

- Understand genetic susceptibility to stroke, as well as to the genetic influence on acute stroke pathology and recovery. 


\section{Conclusion}

The workshop highlighted a number of research priorities for the area of stroke research in Europe. Implicit in some of these priorities identified is the need for reinforced collaboration within the stroke research community. In addition to identifying research priorities, examples of successful European collaborations in stroke research were presented at the workshop. It will be important to build on these experiences in the future. An option could be to create a more formal stroke research network.

\section{Acknowledgements}

We wish to thank the European Union for hosting this workshop. We are indebted to Dr. Virginija Dambrauskaite for her excellent organization of the workshop and her helpful contributions to the workshop summary. We also thank Dr. Elmar Nimmesgern and Dr. Alain Vanvossel for their valuable guidance in this endeavor.

\section{References}

1 Sudlow CL, Warlow CP: Comparable studies of the incidence of stroke and its pathological types: results from an international collaboration. International Stroke Incidence Collaboration. Stroke 1997;28:491-499.

2 Murray CJL, Lopez AD: The Global Burden of Disease: A Comprehensive Assessment of Mortality and Disability from Diseases, Injuries, and Risk Factors in 1990 and Projected to 2020. Boston, Harvard University Press, 1996.

3 Menken M, Munsat TL, Toole JF: The global burden of disease study: implications for neurology. Arch Neurol 2000;57:418-420. 